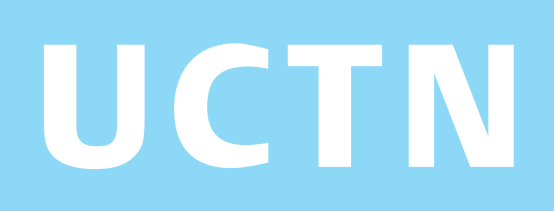

\title{
Invagination of the muscularis propria in a polyp stalk: a rare cause of post-polypectomy perforation of the colon
}
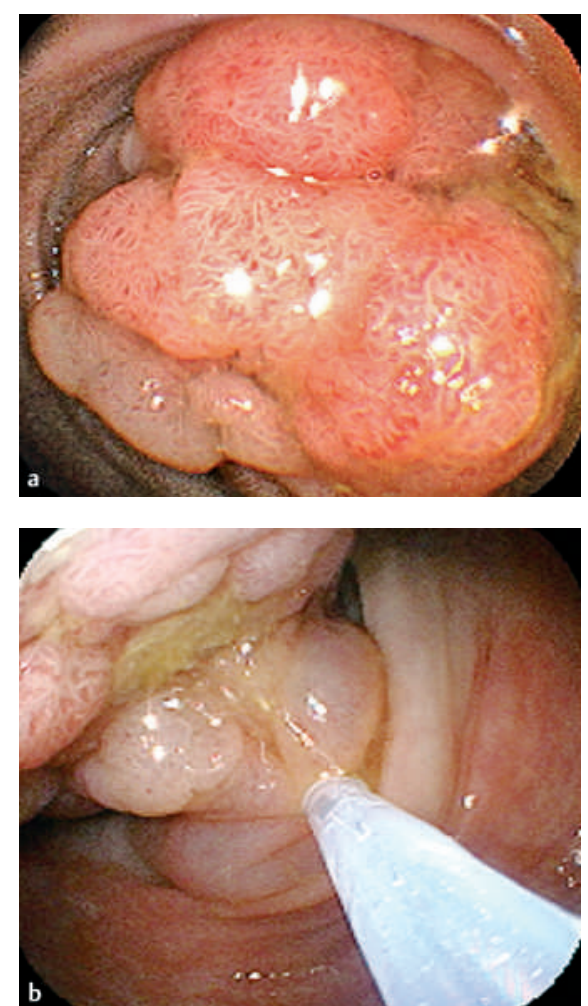

E78

Figure 1 Endoscopic views showing the polyp in the sigmoid colon (a) and the pedicle of the polyp (b).
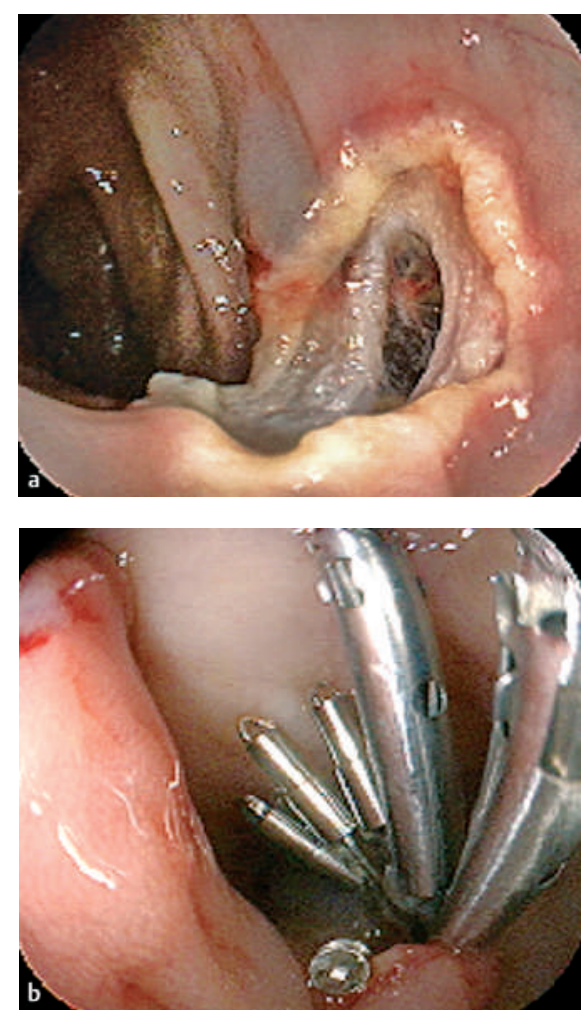

Figure 2 Endoscopic views of the post-polypectomy site, showing the defect before closure (a) and the closure of the perforation with endoclips (b).

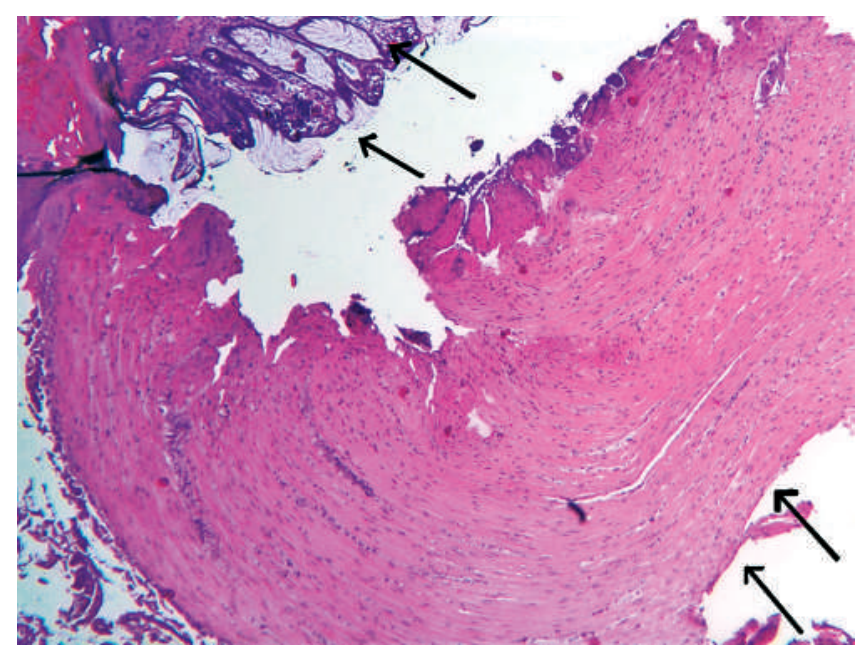

Figure 3 Histological section of the polyp stalk, showing normal colonic glands in the stalk (upper-left-hand corner), and a thick portion of muscularis propria invagination (lower-righthand corner)(hematoxylin and eosin stain, original magnification $\times 40$ ).

A 23-year-old white woman underwent colonoscopy for the investigation of hematochezia. She was found to have a 5$\mathrm{cm}$ pedunculated polyp in the sigmoid colon (Figure 1). A standard injection-assisted polypectomy was performed. A 10$\mathrm{mm}$ defect was noted immediately after- ward at the polypectomy site (Figure $\mathbf{2}$ a), and this was closed using six endoscopic clips (Figure $\mathbf{2} \mathbf{b}$ ). The area was tattooed with India ink.

After the procedure the patient developed severe abdominal pain and leukocystosis.

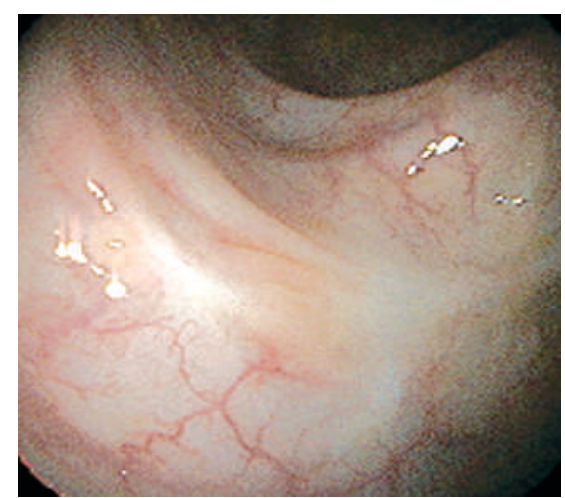

Figure 4 Endoscopic view of the polypectomy site 6 months later.

Computed tomography showed retroperitoneal air. She was managed conservatively, including antibiotics, and was discharged home 5 days later. Histology revealed a tubulovillous adenoma with a thick portion of the muscularis propria invaginated in the stalk (Figure 3). Her follow-up colonoscopy at 6 months was normal (Figure 4).

In general, polyp stalks do not contain a muscularis propria layer. However, removal of the muscularis propria layer is strongly correlated with colon perforation in patients with large colonic lipomas [1]. The exact frequency of this finding in post-polypectomy perforation is unknown. In our patient, intestinal peristalsis probably caused a continuous pulling effect on the polyp and its pedicle, which dragged the attached bowel segment, resulting in mechanical protrusion of the deeper layer of the bowel wall into the polyp stalk. Pedunculated tumors have been reported to act as the leading point in intussusception [2]. There is limited evidence that large lipomas can be removed safely by endoscopy after endosonographically confirming that the muscularis propria layer is not involved [3]. The same approach might apply to large pedunculated polyps with a broad pedicle. Endosonographic evaluation prior to endoscopic removal might identify the pres-

DOI: $10.1055 / \mathrm{s}-2006-945155$ 
ence of a muscularis propria layer in the stalk and could help to avoid the complication of post-polypectomy perforation in this setting.

The use of endoclips to close gastrointestinal perforations has been reported previously [4], based on the premise that immediate closure of the perforation should minimize contamination of the peritoneal cavity. However, controlled data are lacking. We treated our patient conservatively because of the small size of the perforation and because it was closed immediately, thus minimizing the risk of fecal contamination.

\section{Acknowledgment}

This case report was accepted for the American Society for Gastrointestinal Endoscopy (ASGE) Audiovisual Forum, Digestive Disease Week, May 2006, Los Angeles, California, USA.
Endoscopy_UCTN_Code_CPL_1AJ_2AC

\section{S. Ahlawat, F. H. Al-Kawas}

Division of Gastroenterology, Department of Medicine, Georgetown University Hospital, Washington DC, USA.

\section{References}

${ }^{1}$ Pfeil SA, Weaver MG, Abdul-Karim FW, Yang P. Colonic lipomas: outcome of endoscopic removal. Gastrointest Endosc 1990; 36: 435-438

${ }^{2}$ Huang BY, Warshauer DM. Adult intussusceptions: diagnosis and clinical relevance. Radiol Clin N Am 2003; 41: 1137- 1151
${ }^{3}$ Kim CY, Bandres D, Tio TL et al. Endoscopic removal of large colonic lipomas. Gastrointest Endosc 2002; 55: 929-931

${ }^{4}$ Charabaty-Pishvaian A, Al-Kawas F. Endoscopic treatment of duodenal perforation using a clipping device: case report and review of the literature. South Med J 2004; 97: $190-193$

\section{Corresponding author}

\section{F. H. Al-Kawas, MD}

Division of Gastroenterology Georgetown University Hospital 3800 Reservoir Rd., NW Washington DC 20007

USA

Fax: $\quad+1-202-444-0417$

Email: alkawasf@gunet. georgetown.edu 